\title{
Das baskische Zeitwort und Julien Vinson.
}

Wenn ich die Gastfreundschaft dieser romanistischen Zeitschrift als Baskologe in Anspruch nehme, so doch nur für ein Plätzchen, das etwa in demselben Verhältnis zu ihrer weiten Räumlichkeit steht wie die baskische Insel $\mathrm{zu}$ dem sie umschliefsenden romanischen Ozean. Auch handelt es sich zum Teil um Fragen, die dem Verständnis weiterer Kreise zugänglich sind. Julien Vinson hat in der Revue de linguistique XXVII, 97-1 10 meine Studie "Über die Entstehung der Bezugsformen des baskischen Zeitworts" besprochen; ich bin ihm dankbar dafür und insbesondere für die liebenswürdige Form, in die er seinen Widerspruch gekleidet hat. Das Prädikat "qui nisi quod ipse fecit, nil rectum putat" weist er am Schlusse nicht als ein ganz unmögliches von sich ab; und in der That, es kommt uns allen in einem gewissen Grade zu. Wer von uns einmal einen festen Standpunkt eingenommen hat, weicht auch vor schwerem Angriff nicht zurück; wie jene alten tapfern Ritter, scheuen wir das „a loi de recreü“. Die Dinge in Ordnung zu bringen, dazu sind die Schiedsgerichte da, wenn nicht die der Mit-, so die der Nachlebenden. Nur in Einem mülste eine Änderung eintreten; es mürste so etwas wie Kampfregeln beobachtet werden, nämlich zwischen Leuten, die einander für ebenbürtig halten. Wird auf des Einen ausführliche Begründung der eigenen oder Bekämpfung einer fremden Ansicht von dem Andern geantwortet: „bleibt unerwiesen", "hat mich nicht zu überzeugen vermocht" u. s. w., so hat solches entweder den Sinn, dafs ihm Zeit und Lust fehle auf die Sache einzugehen, hat also den Wert einer rein privaten Mitteilung, oder es soll dadurch die Meinung Anderer beeinflufst werden, dann hat es den Wert einer Censur, die der Lehrer dem Schüler erteilt, dann wird eben von jener Voraussetzung der Ebenbürtigkeit abgegangen. Was die Kenntnis von den Thatsachen der baskischen Sprache anlangt, so verhalte ich mich zu Vinson wie der Schüler zum Lehrer; was aber deren wissenschaftliche Deutung, so betrachte ich mich ihm als ebenbürtig. Demnach hätte ich gewünscht, er wäre meinen Gründen mit seinen Gründen begegnet, er wäre, wenn seine Mufse oder der Raum seiner Zeitschrift für mehr nicht ausreichte, wenigstens mit Rücksicht auf den Hauptpunkt, die Entstehung der Bezugsformen, meiner Beweisführung gefolgt. Die paar hingeworfenen Bemerkungen nehme ich auf.

Zunächst habe ich mich in methodischer Hinsicht zu recht- 
fertigen. Vinson sagt: „M. Sch. a soin de déclarer que ce mémoire est extrait d'un travail d'ensemble sur le verbe basque; je regrette, quant à moi, qu'il l'ait rendu public le premier, parce qu'd mon avis, il convient de procéder toujours méthodiquement." Ich habe nicht im entferntesten daran gedacht eine umfassendere Arbeit über das baskische Zeitwort zu veröffentlichen und keine Äufserung gethan, die in diesem Sinne ausgelegt werden könnte. Ich beginne meine Studie mit den Worten: „Unter den Anmerkungen, die ich zu gewissen baskischen Texten niederschreibe, ist eine so in die Breite und Länge gewachsen, dafs ich sie von den andern abtrennen mufs und hier als eigene Abhandlung bringe.“ Weiter heifst es: „Avant ce que M. Sch. appelle les formes de relation, il faut etudier les formes ordinaires, directes." Gewils gilt das im ganzen genommen; dem Einzelnen aber darf nicht verwehrt sein sich auf einem schon vielfach bebauten Gebiete aus bestimmtem Anlafs ein kleineres Feld zur Bearbeitung auszuwählen. Ich habe zudem nicht nur in der Einleitung, sondern im ganzen Verlauf meiner Studie über die meisten wesentlichen und schwierigen Punkte der baskischen Konjugation meine Ansichten mehr oder weniger ausführlich dargelegt. Ich verdiene also den Vorwurf des Hysteronproteron in keiner Weise, am wenigsten von Vinson. Denn er hat uns lange mit der Hoffnung hingehalten, er werde uns ein Gesamtbild dieser Konjugation bieten wie sie vor seinen Blicken steht; wir selbst können es, ohne Lücken und Widersprüche, uns nicht aus den zahlreichen Bemerkungen zusammensetzen, die er seit einem Vierteljahrhundert in den vielen Bänden der Revue de linguistique und an andern Orten verstreut hat. Die mehr zusammenhängenden fallen in die erste Hälfte dieser Periode; in neuerer Zeit scheint Vinsons sprachwissenschaftliches Interesse am Baskischen abgenommen zu haben. Hält er nun, nachdem er selbst und die baskischen Studien und die Sprachwissenschaft überhaupt so beträchtlich fortgeschritten sind, noch heute an der "Gunierung" von Vokalen (wie des $u$ in dut $<$ daut), selbst von „euphonischen" fest, an der „Verstärkung “ des $n$ zu nd in ninduen u. a. (mit Hinweis auf gambara, ondra, die ja span. cambra, hondra entsprechen), an dieser Unmasse von „euphonischen Buchstaben" (z. B. in gachataaz drei solcher a) u. s. w.? Ich glaubte dergleichen nicht ohne weiteres ihm zutrauen zu dürfen. Jetzt allerdings sagt er: "i'élais arrivé d des conclusions que je crois $\grave{a}$ peu près déffnilives", und setzt hinzu: „je les ai rapportées dans plusieurs publications qui paraissent avoir echappe en partie d M. Sch." Auch gegen diesen Vorwurf des mangelhaften Quellenstudiums mufs ich mich wehren. Nicht als ob von dem Vielen, was Vinson an so vielen Stellen vorgebracht hat, mir nicht dies und jenes hätte entgehen können; nur von den als Belegen angeführten sieben Punkten, deren Feststellung er sich beimilst, ist mir kein einziger entgangen. Einige davon lagen aus meinem Wege; so brauchte ich nicht $\mathrm{zu}$ erörtern, ob das Vollpronomen $h i$ aus ${ }^{*} k i$ hervorgegangen ist (wenn ich S. $8 k$ - als "lautliche Variante" von $h$ - be- 
zeichne, so meine ich damit nur die geschichtliche Identität beider schlechtweg), und auch die Zahl der Zeiten (und Modi) interessierte mich nicht weiter, in Betreff deren übrigens die Meinungsverschiedenheit nicht von besonderer Bedeutung ist. Zuek als pleonastischer Plural ergiebt sich Jedem von selbst; so war es ja schon vor Vinson von van Eys 1867 ausdrücklich erklärt worden. Da, wo ich mich über das $-n$ des Imperfekts äufsere (S. 4), habe ich allerdings Vinsons Annahme, dafs es ein "signe conjonctif“, und "le suffixe caractéristique du conjonctif“ (zu Ribáry S. I I I) sei, mit Stillschweigen übergangen; denn ich sehe nicht, dafs der Versuch unternommen worden wäre, diesen hier doch unwahrscheinlichen Charakter des $-\boldsymbol{n}$ durch irgendwelche Hinweise wahrscheinlicher zu machen. Ebenso entziehen sich Vinsons hypothetische Paradigmen des Präsens und Imperfekts von "sein" und "haben“ der Nachprüfung; dafs sie mir bekannt waren, zeigt S. 35, wo ich sein $d a$ aus *diz anführe. In welchem Sinne übrigens *ninu, welches doch der Vorläufer von ninduen, "mich hatte er" und nicht von nuen, ,ich hatte ihn" ist, dem dut, ,ich habe ihn“ statt dem nau, „mich hat er" gegenübergestellt wird, leuchtet mir nicht ein. Vinson knüpft hieran die Bemerkung: "L'interversion de position des pronoms sujets entre le présent et l'imparfait transitifs ne doit pas nous étonner, car on sait qu'elle est normale dans les langues símitiques", und vergleicht arabische Formen. Ich sage S. 4: „Die Erklärung für die auseinandergesetzte Verschiedenheit der Form und Stellung der Pronomina im Präsens und Präteritum kann in nichts anderem gesucht werden als in der von Haus aus nicht rein zeitlichen Bedeutungsverschiedenheit der beiden Verbalstämme“", ebenfails mit Heranziehung arabischer Formen. Damit aber glaubte ich noch nicht die Erklärung gefunden. Wo eine Schwicrigkeit wie die in dem Verhältnis zwischen den Formen nuen und ninduen liegende so leicht verkannt wird, könnte man es sich immerhin zum Verdienst anrechnen diese Schwierigkeit beleuchtet zu haben. Die Erkenntnis, dafs $k i$ das Zeichen des Dativs ist, habe ich S. 29 Vinson gedankt. Seine Meinung, dafs $t z, t z a, t z i, z k i, z k a$ Varianten des pluralischen $z$ seien, habe ich S. $32 \mathrm{f}$. eingehend besprochen und berichtigt. Auch bezieht er sich ja selbst später darauf: "La théorie passive a permis a $M$. Sch. de regarder zka, tza, tzi etc. comme des représentants $d u$ ki datif." Das aber ist nicht richtig, ich erblicke in $t z i$ das einfache Pluralzeichen, in $z k i$ dasselbe + Dativzeichen (S. 32), in $z k a, t z a$ das Pluralzeichen + Dativ der 3. P. (S. 33); die "passivische Theorie“ spielt hier gar nicht mit, der Dativ mufs ja von ihr unberührt bleiben. Vinson fährt fort: „Nous savons qu'en basque $\mathrm{z}$ peut se renforcer en $\mathrm{tz}$, que tz permute avec $\mathrm{zt}$ (ex. bost, bortz "cinq"), que zt peut passer $\grave{a} \mathrm{zk}$ et dans dakitzat ou dakizkit "je les vois" [lies: "je les sais"] nous ne voyons point de datif, mais le pluriel $\mathrm{z}$ du régime direct qui a pris avant le $\mathrm{t}$ un a euphonique et s'est renforcé en $\mathrm{tz}$ puis en $\mathrm{zk}$ tandis que l'a s'affaiblissait en $\mathrm{i}$. M. Sch. ne tient peut-être pas assez compte de la phonétique." Vielleicht zu sehr. 
Ich kann nicht zugeben, dafs $z$ sich zu $t z$ verstärke (dieses schwächt sich zu jenem ab) und dafs $t z$ mit $z t$ aufser nach $r$ wechsele (vgl. Vinson Rev. III, 457), und die Vertretung von $z t$ durch $z k$ läfst sich als rein lautliche nicht erweisen. Wie das mit dem Dativ der 3. P. oder mit dem blorsen Dativzeichen verbundene Pluralzeichen zu einfach pluralischer Bedeutung gelangt, habe ich an jener Stelle auseinandergesetzt.

Als Beispiel der Verschiedenheit zwischen unsern beiderseitigen analytischen Methoden führt Vinson an: zitzazkidaketenan, „sie könnten sie (PI.) mir haben, o Frau". Sie besteht in zweierlei (te erkläre ich ja nicht als "pronom faiseur", sondern als Pluralzeichen für das Urheberpronomen; meine Buchstaben- und Zahlenformel ist in arger Entstellung wiedergegeben worden). Erstens darin, dafs Vinson die drei $a$ als „euphonische“ betrachtet, während ich sie zum vorhergehenden Konsonanten ziehe: $z a$ (,sein"), da (,mir"), na (,Frau!"). Über die „euphonischen Buchstaben“, die in der baskischen Grammatik eine so grofse Rolle spielen, spreche ich mich S. 28 aus; der Standpunkt, von dem man solche erblickt, ist, glaub' ich, in der Sprachforschung überhaupt ein veralteter. Ich frage: "hat sich $a$ zwischen $z-z, d-k, n-n$ aus einem der beiden Konsonanten entwickeln können?“ Ich antworte: „Nein; und es wären $z-z, n-n$ zusammengefallen." Ebenso wenig sind diese $a$ als Fälle lautlicher Analogie denkbar. Und endlich, da $z, d, n$ einmal selbständige Wörter gewesen sind, so müssen sie doch einen Vokal gehabt haben, und warum wäre dies nicht $a$ gewesen? Zweitens setzt Vinson $i t z=$ "sein", ich it als Pluralzeichen, $z a=$ „sein". Auch hierüber können sich die des Baskischen Unkundigen ein gewisses Urteil bilden. Dem zitzazkidaketenan, „sie könnten sie ..." steht zur Seite zizadaketenan, „sie könnten ihn ...". Wenn das erste $t$ der ersteren Form nicht mit zur Pluralbezeichnung gehört, so muissen wir eirien doppelten Stamm annehmen, das eine Mal itza, das andere Mal iza. Und wenn wir nun andere Formen in entsprechender Weise zergliedern, dann bekommen wir noch eine Menge von Stämmen für "sein": eza, atza u. s. w. Wie wäre aber eine solche Vielheit von Stümmen, die Vinson in der That mit andern Baskologen anzunehınen scheint, zu erklären? Aus der lautlichen Ungebung nur zum geringeren 'Teil.

Dals ich mit Fr. Müller die einzige Präteritalform des Baskischen schlechtweg als Präteritum bezeichne, nicht dem Herkommen nach als Imperfekt, will ich nicht weiter verteidigen, obwohl mir die Ursprünglichkeit ihrer imperfektischen Bedeutung keineswegs feststeht. Unsere ganze morphologische Terminologie ist eine so bresthafte, dafs sie wohl durch eine andere ersetzt werden sollte; vorderhand begnügen wir uns damit Mifsverständnisse zu vermeiden. Auch nur zu einem solchen Zweck wählte ich den von Vinson beanstandeten Ausdruck „Bezugsformen“; „Anredeformen" (formes allocutives) ist nicht zu brauchen: „du hast" ist doch gewifs eine „Anredeform“, aber keine „Bezugsform“. 
Wie urteilt nun Vinson über den Kern meiner Studie, die Zurückführung der Bezugsformen auf Zielformen? Das Pronomen der 2. P., das in den ersteren steckt, ist ihm ein Vokativ. Diese Möglichkeit gebe ich ja S. 8.59 von der begrifflichen Seite zu, und Vinson sollte seinerseits mir die Möglichkeit zugeben, dafs es ein Dativ ist. Er sagt aber, ziztak sei nicht, wie ich thue, zu übersetzen: „sie sind mir dir (Mann)“, sondern „sie sind mir, o du (Mann!)"; "on ne voit pas ce que le dalif viendrait faire dans ces formes". Ja, leugnet er denn das Vorhandensein eines Dativus ethicus in andern Sprachen, worauf ich mich ausdrücklich berufen habe? Ist es nicht ebenso natürlich und in den Sprachen überhaupt ebenso häufig zu sagen: „ich habe ihn dir" wie "du! ich habe ihn" und nicht sogar häufiger als „ich habe ihn, du!"“ (was der baskischen Wortstellung entsprechen würde). Die Entscheidung zwischen Dativ und Vokativ ergiebt sich nun aus einem formalen Umstand: dem Bezugspronomen geht in der Regel ein $i$ voraus, das doch keinenfalls den Sinn eines „o!“ haben kann, und das ich dem dativischen $i$ (für älteres $k i$ ) gleichsetze. Über dies $i$ aber, welches die Achse meiner ganzen Untersuchung bildet, äufsert sich Vinson mit keinem Wörtchen. Er sagt von den Bezugsformen: „elles sont formées simplement par l'addition d'un $\mathrm{k}$, d'un $\mathrm{n}$ et même d'un $\mathrm{zu}$ (toi, poli) aux formes ordinaires." Wiederum wende ich mich auch an die Fernerstehenden mit der Frage, ob dik, ziayek, niezakan, $\tilde{n} e u n k e k$ u.s.w. einfach durch Hinzufügung eines $k$ aus $d u$, zaye, nazan, neunke u. s. w. gebildet sind? Beiläufig spricht Vinson bezüglich des auch mir ganz dunkeln weiblichen $n$ die Vermutung aus, es habe verkleinernde, kosende Bedeutung und sei durch $y, \tilde{n}$ aus dem beidgeschlechtigen $k$ entstanden. Nach allem, was ich von baskischem Lautwandel weifs, ist das geradezu unmöglich.

Vinson berührt noch einen andern Punkt, der für die Angliederung des Baskischen an andere Sprachen von höchster Wichtigkeit ist. Dafs die Konjugation des transitiven Zeitworts im Baskischen auf passivische Auffassung zurückweist, ist wohl schon längst geahnt worden (eine mir vorschwebende Stelle habe ich noch nicht wieder auffinden können), und dann von Fr. Müller 1887 , von mir 1888 und von V.Stempf 1890 (also durfte Vinson nicht sagen: „M. Sch. adopte la théorie de M. Stempf") ausgesprochen und schliefslich an der Spitze meiner Studie hervorgehoben worden. Gegen diese, die "passivische“ Theorie, wendet sich nun Vinson. Sie stützt sich auf zwei Umstände: auf die Anordnung der Pronomina (bei den transitiven Zeitwörtern hat das scheinbare Objekt dieselbe Stellung wie bei den intransitiven das Subjekt) - diese läfst Vinson ganz unerörtert - und auf die Kasusbildung. Wir haben zwei Kasusformen, die eine, endungslose, für das „Objekt“ beim transitiven und das Subjekt beim intransitiven, die andere, auf $k$ ausgehende, für das "Subjekt" beim transitiven Zeitwort. Das darf man nicht so ausdrücken wie Vinson thut: "S'il y a deux nominatifs en basque, il n'y a pas d'accusatif“" (S. 97). Wir hätten 
ja dann bei jedem transitiven Zeitwort einen doppelten Nominativ und müfsten einen solchen auch in einem lateinischen Satz wie rubigo consumit ferrum finden. Die Sache ist die. Wir bemerken zunächst zwei Formen (A, B) und zwei Funktionen (a, b), von denen die einen sich mit den andern kreuzen: $A$ a, A b, B b. Nehmen wir nun passive Konstruktion an, so decken sie sich: $\mathrm{AaBb}$, d. i. Subjektskasus und Instrumental. Vinson erklärt das Nebeneinanderbestehen der beiden Nominative, des intransitiven und des transitiven, nicht; ein solches ist doch etwas Ungewöhnliches, und wo es sonst vorkommt, nehmen wir es immer als Zeichen für die passivische Auffassung des transitiven Zeitworts. Er thut recht daran sich nicht auf das Sprachgefühl der heutigen Basken zu berufen, für die ja allerdings dut nichts anderes ist als „j'ai“ oder „je l'ai“; wenn sie übrigens, wie Vinson hierbei anführt, auch im Französischen où avez-vous mon chapeau? für où est mon chapeau? sagen, so handelt es sich da um einen Reflex des ursprünglichen Verhältnisses („gehabt werden" $=$ „sein"). Eine andere bekannte Thatsache, die von Vinson ohne weitere Nutzanwendung erwähnt wird, ist die, dafs duena sowohl heifst "der welcher ihn hat" als „der welchen er hat", worin ich eine Wirkung der aus dem Romanischen ins Baskische eingedrungenen aktivischen Auffassung erblicke. Vinson meint nun, unsere Theorie nötige vorauszusetzen: 1) "que le pronom régime indirect n'est pas exprimé dans la forme de $3^{e}$ personne ce qu'on comprend beaucoup moins que lorsqu'il s'agit du sujel" - für mich ist umgekehrt z. B. „ich werde gehabt [von ihm]" viel wahrscheinlicher als "mich hat [er]", was ich hier nicht begründen will, da auch Vinson seine Meinung nicht begründet; 2) "que les pronoms expriment l'instrumental dans les formes transitives et le datif dans les formes intransitives" - nein, der Dativ ist ursprünglich und meistens noch durch $-k i-,-i-$ gekennzeichnel; übrigens würde ja die Schwierigkeit dieselbe bleiben, wenn wir an Stelle des Instrumentals wieder den Nominativ setzen. „Cette hypothise n'explique point la diffirence remarquable qui existe entre le prtsent et l'imparfait actif en ce qui concerne la position des pronoms" das räume ich ein; aber dieser Widerstreit wird ebenso wenig bei der überlieferten und durch Vinson vertretenen Anschauung aufgehellt, obwohl er auf der vorhergehenden Seite sagt, dafs er "ne doit pas nous étonner", ohne dafür etwas anderes anzuführen als eine gewisse Analogic mit semitischem Sprachgebrauch, die ja ihm nicht günstiger ist als uns (s. oben S. 534). „Enfin les nombreux verbes simples ne se prêtent guère à cetle passivité, si le moi nous est permis" - ich sehe nicht, dals sich in dieser Beziehung "haben" von "thun", "tragen“, „wissen“, „sehen" unterscheide; man erwäge, inwieweit in den beiden letzten Zeitwörtern der Begriff der Aktivität hervortritt. "J'ai peine à saisir l'avantage qu'il y a d traduire dagidazun: 'qu'il soit fait à moi par vous' au lieu de 'que vous me le fassiez'; où prend-on le 'par'? En réalité, il me semble que l'on complique beaucoup une chose déjà compliqué." Hier stellt sich Vinson 
ganz auf den Standpunkt des Utilitariers und des Franzosen. Was in einer Übersetzung verwickelt erscheint, das ist es doch nie für den Basken gewesen. Warum soll denn par im Baskischen überhaupt ausgedrückt sein? Der Instrumental wird durch seine Stellung vom Subjektskasus und der Dativ von ihm durch sein Vorzeichen geschieden. Dem Franzosen ist vous aimez Marie durchaus klar; wenn nun der Spanier, welcher sagt: $V$. quiere $a$ María, fragen wollte, wo im französischen Satze das $a$ stecke? So klar wie dem Franzosen sein vous aimez Marie ist dem Basken sein Marie est aimée vous.

Am Schlusse sagt Vinson, er habe viele andere Bemerkungen über meine Arbeit $\mathrm{zu}$ machen. Fr macht noch eine, übersieht jedoch, dafs es sich dabei wieder um nichts anderes handelt als um die vorher von ihm besprochene „passivische" Theorie. Wer ihr huldigt, kann die Worte: astoa ..., bere lagunak utzirik, lehoiñ larruz bestiturik nicht anders übersetzen als ich gethan habe. Doch betrachten wir den Fall einmal von Vinsons Scitc aus. Utzen heifst „verlassen“, bestilzen „bekleiden", utzi „verlassen“ (P\%.), bestitu „bekleidet", entsprechende Bedeutung haben die gerundischen Formen. Wenn nun „utzirik et bestiturik joucnt le même rôle dans cette phrase", wie kann man jenes mit ",ayant quitté" und dies mit "s'étant revêtu“" übersetzen, d. h. das eine aktiv, das andere passiv oder reflexiv nehmen? Astoa gehört zwar zu goan zen, wie Vinson sagt, und auch zu bestiturik, aber neben ulzirik mufste jedenfalls astoak stehen. Die französische Übersetzung: „l'âne, ayant quitté ses compagnons, revêtu d'une peau de lion, alla" verdeckt die Schwierigkeit; das Baskische ist in der That verwickelter, es verlangt hier die beiden Nominative, den transitiven und den intransitiven, und der erstere ist verschwiegen worden. Für mein „relictis sociis" meint Vinson „de même que pour rendre 'alandonné par ses compagnons' il faudrait dire lagunek utzirik", wo er utzirik, das er vorher als ,ayant quitté", also aktiv, genommen hat, als „abandonné“, also passiv, nimmt. Dafs aber lagunck utzirik auch die Bedeutung habe: „relictis sociis", also „les compagnons étant abandonnés", das möchte ich von Vinson wiederhoit sehen, ehe ich mich darüber äufsere.

Vinson will auf seine Bemerkungen und Ausstellungen zurückkommen, wenn die Fortsetzung oder vielmehr der Abschlufs meiner Arbeit vorliege. Wie es damit steht, ist schon oben gesagt worden. Meine Studie bietet Vinson Gelegenheit, meine Ansichten über die verschiedensten Punkte zu prüfen und auch, woran mir vor allem liegt, über manche von mir ausdrücklich bezeichneten Partien, wie die Gruppe der „unpersönlich-transitiven“ Zeitwörter (S. 39-44), thatsächliche Auskünfte zu geben, wozu er wie kein Zweiter befähigt ist. Nun aber mufs ich ihn bitten, zunächst auf meine Gegenbemerkungen und Fragen zu antworten; denn es ist doch wichtiger, dafs Weniges gründlich durchgesprochen, als dafs Vieles gestreift werde.

H. SChuchardT. 